\title{
Estratégias de aprendizagem empregadas por estudantes do Ensino Fundamental
}

\author{
Manuela Ramos Caldas Lins \\ Monilly Ramos Araujo \\ Carla Alexandra da Silva Moita Minervino
}

\begin{abstract}
Resumo
Analisa-se o repertório de estratégias de aprendizagem utilizado por escolares da cidade de Campina Grande, PB. Participaram do estudo 491 estudantes, de ambos os sexos, matriculados do $6^{\circ}$ ao $9^{\circ}$ ano do Ensino Fundamental de escolas particulares e públicas, com idade média de 12 anos e 7 meses $(D P=1,7)$. Os resultados foram obtidos mediante a aplicação coletiva de uma escala de Estratégias de Aprendizagem e indicaram haver diferença no que concerne à utilização de estratégias por alunos repetentes e não repetentes, bem como quando comparados os grupos tomando por base a autopercepção do seu desempenho, sexo, idade, ano cursado e tipo de escola. Tais resultados sugerem que os estudantes podem ter consciência do uso efetivo das estratégias de aprendizagem, uma vez que as utilizam regularmente em seu cotidiano. Sugere-se, pois, que novos estudos sejam realizados visando aprofundar os resultados ora apresentados.
\end{abstract}

Palavras-chave: Estratégias de aprendizagem, metacognição, ensino fundamental.

\section{Learning strategies used by a group of children of primary school}

\begin{abstract}
In this work, we analyze the repertoire of learning strategies used by schoolchildren of the city of Campina Grande, Paraiba, Brazil. The study included 491 students of both sexes enrolled in the 6 th to 9 th year of elementary school for private and public schools, with an average age of 12 years and 7 months $(S D=1.7)$. The results were obtained by applying a collective scale of Learning Strategies. They indicated that there is a difference regarding the use of strategies by repeating and non repeating students. The difference is visible too, when we study groups according to gender, age, year and type of school attended. These results suggest that students may be aware of effective use of learning strategies, since they use them regularly in their daily lives. Our research may suggest that further studies are needed in order to deepen and broaden the perception of the results presented here.
\end{abstract}

Keywords: Learning strategies, metacognition, elementary school.

\section{Estrategias de Aprendizaje utilizadas por estudiantes de Educaciõn Básica}

\section{Resumen}

Se analiza el repertorio de estrategias de aprendizaje utilizado por escolares de la ciudad de Campina Grande, Paraíba. Participaron del estudio 491 estudiantes, de ambos sexos, matriculados del $6^{\circ}$ al $9^{\circ}$ año de Educación Básica de escuelas particulares y públicas, con edad promedio de 12 años y 7 meses $(D P=1,7)$. Los resultados se obtuvieron mediante aplicación colectiva de una escala de Estrategias de Aprendizaje e indicaron que hay diferencia en lo concerniente a la utilización de estrategias por alumnos repitientes y no repitientes, así como cuando se comparan los grupos tomando por base la autopercepción de su rendimiento, sexo, edad, año cursado y tipo de escuela. Tales resultados sugieren que los estudiantes pueden tener conciencia del uso efectivo de estrategias de aprendizaje, una vez que las utilizan regularmente en su cotidiano. Se sugiere que sean realizados nuevos estudios con el objetivo de profundizar los resultados aquí presentados.

Palabras Clave: Estrategias de aprendizaje, metacognición, educación básica. 


\section{Introdução}

Pesquisas nacionais (Almeida, 2002; Boruchovitch, 1999) demonstraram que intervenções pedagógicas e psicopedagógicas podem ser eficazes para evitar o fracasso escolar. Tais intervenções podem diminuir os índices de repetência e evasão escolar na medida em que proporcionam o ensino de estratégias de aprendizagem. Estas vêm sendo cada vez mais estudadas pelos teóricos da Psicologia Cognitiva baseada no Processamento da Informação, visto que esta se mostra mais aberta a indagações sobre estratégias cognitivas e metacognitivas, processos mentais e representação da informação na memória (Cerqueira, 2000).

Danserau (1985, Nisbett \& Shucksmith, 1987, citados por Pozo, 1996; Pozo, Monereo, \& Castelló, 2004) concebe as estratégias de aprendizagem como sequências integradas de procedimentos ou atividades que se elegem com o propósito deliberado de facilitar a aquisição, o armazenamento e a utilização da informação. Dembo (1994) define as estratégias de aprendizagem como métodos que os estudantes utilizam para adquirir conhecimento. Silva e Sá (1997), por sua vez, acreditam que as estratégias de aprendizagem são processos conscientes, esquematizados pelos estudantes para atingirem objetivos de aprendizagem, sendo, por essa razão, processos controláveis e que podem facilitar realizações específicas.

Cruvinel e Boruchovitch (2004) identificam várias classificações e tipos de estratégias de aprendizagem. Deste modo, Dansereau e cols. (1979) acreditam que as estratégias de aprendizagem devem ser entendidas sob dois aspectos: estratégias primárias e estratégias de apoio. As estratégias primárias propõem-se a ajudar o aluno a organizar, elaborar e integrar a informação, enquanto que as estratégias de apoio são responsáveis pela manutenção de um estado interno satisfatório que favoreça a aprendizagem.

Weinstein e Mayer (1985, citado por Boruchovitch, 1999, 2001; Costa, 2000; Muneiro, 2008), diferentemente, identificaram cinco tipos de estratégias de aprendizagem, organizadas posteriormente: estratégias de ensaio (envolvem repetir ativamente o material a ser aprendido); estratégias de elaboração (implicam na realização de conexões entre o material novo a ser aprendido e o material antigo e familiar); estratégias de organização (referem-se à imposição de estrutura ao material a ser aprendido); estratégias de monitoramento (implicam que o indivíduo deve estar constantemente monitorando a sua capacidade de captar e absorver o conteúdo que está sendo ensinado); e estratégias afetivas (referem-se à eliminação de sentimentos desagradáveis, que não condizem com a aprendizagem).

Outra classificação é a de Pozo (1996), que apresenta as estratégias associativas e de reestruturação. A aprendizagem por associação abarca as estratégias de processamento superficial da informação, à semelhança das estratégias de ensaio citadas anteriormente, enquanto que a aprendizagem por reestruturação demanda estratégias de processamento mais profundo da informação, como, por exemplo, as estratégias de elaboração e as de organização.
Zimmerman e Martinez-Pons (1986), por sua vez, encontraram 14 tipos de estratégias: autoavaliação, organização e transformação, estabelecimento de metas e planejamento, busca de informação, registro de informação, automonitoramento, organização do ambiente, busca de ajuda e revisão, ensaio e memorização e, por fim, revisão de notas.

As estratégias de aprendizagem podem ser tomadas, ainda, como cognitivas ou metacognitivas. Para Dembo (1994), as estratégias cognitivas referem-se a comportamentos e pensamentos que influenciam o processo de aprendizagem de maneira que a informação possa ser armazenada de forma mais eficiente, enquanto que as estratégias metacognitivas são procedimentos que o indivíduo usa para planejar, monitorar e regular o seu próprio pensamento. Verifica-se que, diante das várias classificações propostas, os resultados são variados e é necessário pontuar que o presente trabalho adota esta última linha de pensamento explicitada.

Dembo (1994) afirma que as estratégias de aprendizagem têm a finalidade de auxiliar o estudante no armazenamento e posterior recuperação das informações na memória. De acordo com Muneiro (2008), o emprego adequado das estratégias contribui para expandir o armazenamento e melhorar a recuperação da informação, bem como a capacidade de usá-la eficazmente de acordo com os objetivos propostos. Por essa razão, faz-se necessário orientar os alunos para uma utilização mais adequada das estratégias de aprendizagem.

A diversidade teórica quanto ao conceito de estratégias se manifesta na multiplicidade de instrumentos disponíveis para avaliá-las, como, por exemplo, a Self-Regulated Learning Interview Schedule (SRLIS) de Zimmerman e Martinez-Pons (1986), o Motivated Strategies for Learning Questionnaire (MSLQ) de Pintrich, Smith, Garcia e McKeachie (1991) e o Learning and Study Strategies Inventory (LASSI) de Weinstein e Palmer (2002). No contexto nacional, muitos esforços têm sido realizados para desenvolver instrumentos confiáveis direcionados a mensurar as estratégias de aprendizagem. Nessa direção, Boruchovitch e Santos, em 2001, elaboraram uma proposta de escala, embasada nos instrumentos internacionais, destinada a avaliar as estratégias de aprendizagem utilizadas por universitários e, posteriormente, desenvolveram outra escala para mensurar as estratégias utilizadas por alunos do Ensino Fundamental.

Gomes (2002) aplicou esta escala de estratégias de aprendizagem, composta inicialmente por 40 itens, em conjunto com outros instrumentos, em crianças do $5^{\circ}$ ano do Ensino Fundamental de uma escola pública da cidade de São Paulo. Cruvinel (2003) também a utilizou numa amostra de escolares dos $4^{\circ}, 5^{\circ}$ e $6^{\circ}$ anos de uma escola pública da cidade de Campinas. Ambos os estudos indicaram que as meninas obtiveram uma pontuação superior em relação aos meninos, o que evidencia que as meninas utilizaram um número maior de estratégias de aprendizagem.

Boruchovitch e cols. (2006), visando analisar as propriedades psicométricas da escala de estratégias de aprendizagem, desenvolveram dois estudos. No primeiro, 
305 alunos do $3^{\circ}$ ao $9^{\circ}$ ano do Ensino Fundamental foram entrevistados. Eles responderam às questões traduzidas e adaptadas da Self-Regulated Learning Interview Schedule (Weinstein \& Palmer, 2002). As respostas foram tratadas por meio da análise de conteúdo, quando juízes independentes avaliaram o processo de categorização com concordância de $90 \%$. Com esse resultado, pode-se mapear as estratégias relatadas pelos participantes, bem como foi possível a elaboração de 40 itens que comporiam a versão original da escala.

No segundo estudo, 433 estudantes do $4^{\circ}$ ao $9^{\circ}$ ano de escolas públicas de Campinas e de Catalão (Goiás) responderam à versão da escala com 40 itens. As alternativas de respostas foram dispostas em escala likert de três pontos, mostrando a frequência (sempre, às vezes ou nunca) com que o estudante recorria à estratégia. $\mathrm{A}$ análise fatorial de componentes principais e rotação varimax mostrou a existência de três fatores, com itens apresentando saturação em mais de um fator e/ou carga fatorial menor que 0,35 , o que caracterizou a necessidade de redução da escala para 20 itens. Os três fatores encontrados foram: fator 1 (ausência de estratégias), fator 2 (estratégias cognitivas) e fator 3 (estratégias metacognitivas).

Em um estudo sobre as propriedades psicométricas da escala de 20 itens, Oliveira, Boruchovitch e Santos (2006, citado por Oliveira, 2008) contaram com 206 estudantes de $8^{\circ}$ e $9^{\circ}$ anos do Ensino Fundamental de escolas públicas e privadas. A análise fatorial indicou uma estrutura de três fatores. Os itens agruparam-se em três fatores, tal como o estudo inicial. Observou-se que três itens da escala não discriminaram o fator ao qual pertenciam, por não alcançarem saturação suficiente, em nenhum dos fatores. Os resultados de consistência interna mostraram que o instrumento apresentava índices aceitáveis. Contudo, o estudo confirmou também a necessidade de se ampliar a quantidade de itens da escala, especialmente no que se refere à subescala de estratégias metacognitivas.

Boruchovitch, Oliveira e Santos (2007) realizaram outro estudo sobre a escala de estratégias de aprendizagem com 262 alunos de $5^{\circ}$ ano de escolas públicas da cidade de Campinas, que responderam à versão de 20 itens da escala. Os resultados confirmaram a estrutura de três fatores. Entretanto, os valores da consistência interna da escala como um todo e de cada um dos fatores foram inferiores aos encontrados na amostra de alunos do $8^{\circ}$ e $9^{\circ}$ anos e ligeiramente superiores aos do primeiro estudo.

Diante desses resultados e visando aprimorar a referida escala, Oliveira (2008) construiu e validou uma nova versão da escala na qual acrescentou 17 itens, contando com um total de 37 itens. Participaram 815 estudantes, do $3^{\circ}$ ao $9^{\circ}$ ano do Ensino Fundamental, de ambos os sexos, de escolas públicas e privadas dos estados de São Paulo e Minas Gerais. A análise fatorial indicou uma estrutura de três: Fator 1- ausência de estratégias, com 13 itens (exemplo: você costuma se esquecer de fazer o dever de casa?); Fator 2 - estratégias cognitivas, com 11 itens (exemplo: você costuma grifar as partes importantes do texto para aprender melhor?); e o Fator 3 - estratégias metacognitivas, com 7 itens (exemplo: quando você estuda, costuma perceber que não está entendendo aquilo que está estudando?). Ressalta-se que a escala ficou reduzida a 31 itens, e o alpha de Cronbach foi de 0,79 , revelando que o instrumento apresentava índices aceitáveis de confiabilidade. No presente estudo, utilizou-se esta versão da escala, uma vez que é a mais recente e a que, sob o ponto de vista da análise estatística, apresentou resultados mais satisfatórios.

Com base nesses dados e tendo em vista que as estratégias de aprendizagem influenciam o êxito e a qualidade da aprendizagem, este trabalho objetivou investigar quais estratégias de aprendizagem estão sendo empregadas por alunos do Ensino Fundamental de escolas particulares e públicas da cidade de Campina Grande, interior da Paraíba. Pretendeu-se verificar também a existência de diferenças no uso das estratégias no que se refere ao desempenho escolar autopercebido, à repetência, ao sexo, aos anos escolares e aos tipos de escola dos participantes.

\section{Método}

\section{Aspectos éticos}

Para operacionalização da pesquisa, o projeto foi submetido à apreciação do Comitê de Ética em Pesquisa do Centro de Ciências Biológicas e da Saúde da Universidade Estadual da Paraíba, que emitiu parecer favorável à sua realização sob a CAAE - 0546.0.133.000-08. A presente pesquisa respeitou os padrões éticos de conduta, conforme a Resolução n 196/96 do Conselho Nacional de Saúde/MS.

\section{Participantes}

O estudo foi realizado com 491 alunos matriculados no $6^{\circ}(23,4 \% ; n=115), 7^{\circ}(24,0 \% ; n=118), 8^{\circ}(26,9 \% ; n=132)$ e $9^{\circ}(25,7 \% ; n=126)$ ano do Ensino Fundamental de escolas particulares (55\%; $n=270)$ e públicas $(45 \% ; n=221)$ da cidade de Campina Grande. O sexo feminino representou 52,3\% $(n=257)$ da amostra e o masculino, 47,7\% $(n=234)$. A média de idade foi de 12 anos e 7 meses $(D P=1,7)$, sendo a idade máxima de 18 anos e a mínima de 9 anos.

\section{Instrumento}

O instrumento utilizado para a coleta de dados foi uma "Escala de Estratégias de Aprendizagem", de autoria de Boruchovitch e Santos, validada por Oliveira, em 2008, a qual se caracteriza por:

a) Ficha de identificação dos estudantes - trata-se de um conjunto de questões relativas aos dados pessoais, como nome, sexo, idade, escola e série, além de duas questões. A primeira solicita que o aluno autoavalie o seu desempenho escolar e conta com cinco categorias de resposta - muito 
bem, bem, regular, mal, muito mal. A segunda questiona se o estudante já repetiu alguma série e possui duas alternativas de resposta, a saber: sim ou não.

b) Dados relativos à utilização de estratégias de aprendizagem, contendo 31 questões, com três possibilidades de respostas para cada uma das questões. As categorias são descritas como: sempre, valendo 3 pontos; às vezes, 2 pontos; e nunca, valendo 1 ponto. Os itens pertencentes ao fator ausência de estratégias $(3,7,8,12,15,19,21,23,24$, $25,26,28$ e 30 ) têm sua pontuação invertida. A pontuação máxima possível é 93 pontos e a mínima é 31 pontos.

\section{Procedimentos}

Foram selecionadas por conveniência quatro escolas, sendo duas particulares e duas públicas, dentre as instituições de ensino da cidade. Em seguida, foi realizado o contato com a direção das escolas visando à anuência e consentimento institucional, bem como a definição do cronograma de atividades em comum acordo com a pesquisadora. Selecionaram-se as turmas que comporiam a amostra com base na acessibilidade do professor que estaria ministrando aula nos dias de coleta de dados, para então os Termos de Consentimento Livre e Esclarecido serem distribuídos e devidamente assinados pelos responsáveis legais dos alunos. Nos dias e horários previamente agendados, a pesquisadora compareceu às instituições, e a aplicação da escala de Estratégias de Aprendizagem realizou-se de forma coletiva, com tempo de aproximadamente 20 minutos em cada uma das salas de aula. Em todas as ocasiões, os professores permaneceram no local acompanhando a atividade e, para evitar o vazamento acidental de informações que pudessem vir a comprometer os participantes, a coleta de dados foi realizada pela própria pesquisadora.

\section{Resultados}

Os dados foram tabulados com o auxílio do pacote estatístico Statistical Package for the Social Sciences (SPSS), versão 17 for Windows, e submetidos às estatísticas descritiva e inferencial, visando atender os objetivos propostos. Cabe dizer que, como a distribuição da população foi normal, utilizou testes paramétricos, e o nível de significância adotado foi de 5\%.

Inicialmente, realizaram-se análises referentes às pontuações máximas e mínimas alcançadas na escala de estratégias. No que concerne à pontuação geral, os resultados indicaram que a média obtida pelos participantes na escala foi 68,00 pontos $(D P=8,10)$. O valor mínimo foi 38 pontos e o máximo, 89 pontos, e, considerando a média de pontos dos participantes na escala de estratégias, verificou-se que 254 participantes $(51,8 \%)$ apresentaram escores acima da média, enquanto que 19 participantes $(3,9 \%)$ ficaram na média e 218 alunos (44,3\%), abaixo da média. As pontuações nas subescalas ausência de estratégias, estratégias cognitivas e estratégias metacognitivas também foram levantadas. As médias de pontos foram de 28,90 (DP=4,8), 22,19 (DP=4,2) e 16,92 $(D P=2,2)$, respectivamente.

Quando analisadas as estratégias utilizadas, percebeu-se que a estratégia mais referida pelos participantes, com $81,1 \%$, foi uma estratégia metacognitiva (item 18). Outra estratégia bastante citada refere-se à compreensão de leitura (item 27), sendo que $71,1 \%$ dos participantes revelaram que costumam perceber quando não entenderam a leitura e normalmente param e leem novamente. Quanto à frequência de uso das estratégias de aprendizagem, a maioria das estratégias foi escolhida pelos participantes como sendo empregadas apenas "às vezes".

Nesse sentido, verificou-se que $41,8 \%$ dos participantes admitiram que costumam sentir-se nervosos quando estão fazendo uma tarefa difícil (item 21), enquanto que $61,5 \%$ disseram que se sentem cansados nas atividades escolares (item 12) e $66 \%$ costumam esquecer de fazer o que a professora solicita (item 25). Algumas estratégias mencionadas como sendo utilizadas "sempre" merecem destaque. Cerca de $16,5 \%$ fazem o dever de última hora (item 3), $9 \%$ costumam desistir quando a tarefa é difícil ou chata (item 7) e $8,8 \%$ costumam pensar em outra coisa quando a professora está explicando matéria nova (item 8).

Embora as estratégias de aprendizagem tenham sido mencionadas pela maioria como sendo utilizadas "às vezes", algumas delas, nunca usadas por determinados participantes, merecem ser consideradas. Por exemplo, 29,5\% admitem não ter o costume de ler outros textos sobre o assunto que o professor explicou (item 5), 24,4\% dos participantes não criam perguntas e respostas sobre o assunto que estão estudando (item 20) e 14,3\% nunca pedem ajuda quando têm dúvidas (item 31).

Com relação ao desempenho autopercebido, os resultados indicaram que a maior parte da amostra (96,3\%; $n=473$ ) apresentou uma autopercepção predominantemente positiva acerca do próprio desempenho escolar. Poucos $(3,7 \% ; n=18)$ foram aqueles que demonstraram uma autopercepção negativa.

No que concerne ao índice de repetência dos estudantes, faz-se necessário ressaltar que a amostra não foi equivalente, sendo a quantidade de não repetentes superior à de repetentes. Diante disso, antes das devidas análises, optou-se por selecionar aleatoriamente grupos com a mesma quantidade de participantes com o intuito de verificar se haveriam diferenças relativas à média de pontos na escala. Como não houve diferenças significativas entre os grupos, analisaram-se todos os participantes. Assim, utilizando-se o teste $t$ de Student, percebeu-se que houve diferença significativa $(t=-3,838 ; p<0,001)$ no que se refere à utilização de estratégias de aprendizagem por alunos repetentes e não repetentes, sendo que essa diferença deu-se a favor dos não repetentes, tendo estes alcançado uma média de pontos de 68,91 ( $\mathrm{DP}=8,02)$, enquanto que os repetentes fizeram, em média, 65,88 pontos (DP=7,92).

Analisada a influência da variável sexo, o teste $t$ demonstrou existir diferença estatisticamente significativa entre 
Tabela 1. Distribuição das médias de pontos e desvio padrão por subescalas de acordo com o ano cursado.

\begin{tabular}{|c|c|c|c|c|c|c|}
\hline & \multicolumn{2}{|c|}{ Estratégias cognitivas } & \multicolumn{2}{c|}{$\begin{array}{c}\text { Estratégias } \\
\text { metacognitivas }\end{array}$} & \multicolumn{2}{c|}{ Ausência de estratégias } \\
\cline { 2 - 7 } & $\begin{array}{c}\text { Média de } \\
\text { pontos }\end{array}$ & DP & $\begin{array}{c}\text { Média de } \\
\text { pontos }\end{array}$ & DP & $\begin{array}{c}\text { Média de } \\
\text { pontos }\end{array}$ & DP \\
\hline $6^{\circ}$ ano & 22,83 & 3,9 & 16,47 & 2,3 & 30,37 & 5,5 \\
\hline $7^{\circ}$ ano & 22,25 & 4,2 & 16,70 & 2,2 & 29,00 & 4,6 \\
\hline $8^{\circ}$ ano & 22,60 & 4,2 & 17,20 & 2,0 & 29,19 & 4,5 \\
\hline $9^{\circ}$ ano & 21,11 & 4,4 & 17,23 & 2,2 & 27,17 & 4,2 \\
\hline
\end{tabular}

os grupos na pontuação total da escala $(t=3,386 ; p=0,001)$. De modo geral, as meninas alcançaram uma pontuação maior $(M=69,17)$ do que os meninos $(M=66,72)$. No que concerne às estratégias cognitivas, as meninas obtiveram uma média de 23,03 pontos $(D P=3,9)$ e os meninos, 21,26 pontos $(D P=4,3)$. Nas estratégias metacognitivas, as meninas alcançaram uma média de 17,26 pontos $(D P=2,0)$ e os meninos, 16,53 pontos $(D P=2,3)$. Já no tocante à ausência de estratégias, as meninas fizeram 28,88 pontos $(D P=4,8)$ e os meninos, 28,93 pontos ( $D P=4,8)$.

Quanto à idade, devido à diversidade da amostra, optou-se por agrupar os alunos em dois grupos, o primeiro composto por alunos de 9 a 13 anos e o segundo, por alunos de 14 a 18 anos. $O$ grupo 1 apresentou uma média de idade de 11 anos e 8 meses, enquanto que o grupo 2 , uma média de 14 anos e 5 meses. $O$ teste $t$ demonstrou existir diferença estatisticamente significativa entre os grupos na pontuação total da escala $(t=3,297 ; p=0,001)$. Os resultados indicaram que o grupo 1 obteve, em média, 68,80 pontos $(\mathrm{DP}=8,26)$ e o grupo 2, uma pontuação média de 66,18 ( $\mathrm{DP}=7,46)$, logo o grupo 1 alcançou médias de pontos superiores ao grupo 2. Quando comparados os grupos tomando por base as subescalas, percebeu-se o grupo 1 saiu-se melhor em todas as subescalas analisadas.

Quando analisado o uso das estratégias nos diferentes anos escolares, os dados revelaram haver diferenças estatisticamente significativas $(F=6,905 ; p=0,000)$ entre os grupos. Assim sendo, foi possível verificar que os alunos do $6^{\circ}$ ano obtiveram a melhor pontuação $(M=69,75)$, sendo seguidos pelos alunos do $8^{\circ}$ ano $(M=68,98)$, dos alunos do $7^{\circ}$ ano $(67,95)$ e, por fim, ficaram os alunos do $9^{\circ}$ ano $(M=65,43)$. No que concerne às estratégias cognitivas, as estratégias metacognitivas e a ausência de estratégias os resultados podem ser vistos na tabela 1 .
Por fim, foram analisados os tipos de escola e as estratégias de aprendizagem. Encontraram-se diferenças estatisticamente significativas $(t=1,958 ; p=0,051)$ no que diz respeito à média de pontos obtidas pelos estudantes das escolas particulares e das públicas, sendo que os alunos das primeiras obtiverem escores mais elevados $(M=68,65)$ que os da segunda $(M=67,21)$.

\section{Discussão}

Analisando os resultados, percebeu-se que a maioria dos alunos reporta utilizar estratégias cognitivas e metacognitivas durante a realização de tarefas escolares e de estudo em casa. Nesta perspectiva, Silva e Sá (1997) apontam que o uso adequado das estratégias de aprendizagem permite ao estudante adquirir, organizar e reter as informações necessárias ao processo de aprendizagem e, desta forma, contribuir para o sucesso escolar. De acordo com Oliveira (2008), as estratégias facilitam a aprendizagem, uma vez que possibilitam aos alunos o envolvimento em seu próprio processo de aprendizagem e a consequente diversificação das formas de estudo.

No que concerne às estratégias mais utilizadas, os resultados indicam que os estudantes percebem quando não entendem algum conteúdo, entretanto nem todos conseguem, com base nessa percepção, modificar seu repertório de estratégias. Nesta medida, Silva e Sá (1997) afirmam que, muitas vezes, os estudantes utilizam determinadas estratégias mesmo quando elas são pouco eficazes. Pode-se supor, a partir disso, que os estudantes, ao adotarem determinadas rotinas, não conseguem desvencilhar-se delas porque é difícil modificar hábitos enraizados e, para fazê-lo, é preciso que novos comportamentos sejam aprendidos através do ensino sistemático. 
Outro dado é que a maior parte dos estudantes revelou fazer uso das estratégias apenas "às vezes", o que demonstra que eles, apesar de saberem quais estratégias devam utilizar, não o fazem com a frequência adequada, porque não entendem o significado e importância dessas estratégias para a aprendizagem. Além disso, chama a atenção o fato de alguns estudantes não utilizarem "nunca" determinadas estratégias e tal fato pode ser explicado a partir de duas hipóteses: ou eles desconhecem as estratégias e, consequentemente, não sabem como aprender de maneira eficaz, ou eles conhecem as estratégias e, por não perceberem sua importância, não as utilizam. De acordo com Silva e Sá (1997), para que os estudantes sejam bem sucedidos, precisam compreender a utilidade das estratégias de aprendizagem. Assim sendo, uma opção para reverter o quadro seria, além de mostrar aos estudantes quais as estratégias que podem auxiliá-los, explicar como elas interferem positivamente no processo de aprendizagem.

No que se refere à autopercepção do desempenho, os dados revelaram que a maior parte dos estudantes acredita estar saindo-se muito bem na escola, independente do seu rendimento ou índice de repetência. Tal fato demonstra que muitos alunos não percebem o seu desempenho de maneira fidedigna, ou seja, escolares com problemas de rendimento e de repetência, ao afirmarem estar "muito bem", respondem a essa questão com base em uma convenção social e não na realidade concreta vivida por eles. Nesse sentido, Costa (2000) acredita que o aluno reportar o uso de estratégias de aprendizagem não significa que ele as utilize. Gomes (2002), na mesma perspectiva, afirma que sempre fica a dúvida se os sujeitos respondem ao que eles pensam ser a expectativa do pesquisador ou se as respostas refletem um juízo sobre determinada ação ou a ação real. Por essa razão, Oliveira (2008) aponta que uma autopercepção do desempenho positiva não pode ser considerada sinônimo do emprego de estratégias no momento do estudo.

Analisando o índice de repetência, percebeu-se que há uma diferença estatisticamente significativa entre os grupos, tendo os alunos não repetentes obtido escores mais elevados. Tal fato era esperado, visto que, segundo a literatura, estudantes que utilizam estratégias de aprendizagem tendem a obter melhores resultados e um desempenho superior. Tais resultados também foram encontrados por Cruvinel (2003), que, em sua pesquisa, constatou que os não repetentes tiveram um desempenho levemente superior ao dos repetentes na escala de estratégias de aprendizagem.

No que concerne ao sexo dos respondentes, analisando a pontuação geral na escala de estratégias de aprendizagem, foi verificada uma diferença significativa entre os sexos, tendo as meninas apresentado uma média de pontos maior do que a dos meninos. Quando se analisou o sexo em relação aos anos escolares, ficou evidente que, em todos os anos, nas subescalas de estratégias cognitivas e metacognitivas, as meninas tiveram uma pontuação melhor do que os meninos. Apenas na subescala ausência de estratégias, os meninos dos $8^{\circ}$ e $9^{\circ}$ anos obtiveram uma média de pontos maior que as meninas. Tal dado corrobora os achados de
Oliveira (2008) que, utilizando a escala de 31 itens, encontrou uma diferença estatisticamente significativa entre os sexos na pontuação da referida escala, tendo as meninas se sobressaído. Diferentemente, Cruvinel (2003), com a escala de 40 itens, não encontrou diferença estatisticamente significativa entre os grupos citados, apesar de a média obtida pelas meninas na escala de estratégias de aprendizagem ter sido um pouco superior à média dos meninos.

Quanto à idade, o grupo dos estudantes mais novos alcançou escores superiores quando comparados ao grupo dos mais velhos em todas as subescalas da escala de estratégias de aprendizagem. Oliveira (2008) encontrou resultados semelhantes, exceto no que se refere às estratégias metacognitivas, em que os estudantes mais velhos se sobressaíram. No que se refere às subescalas de estratégias cognitivas e ausência de estratégias, os resultados encontrados estão dentro do esperado, visto que estas estratégias são primárias e requerem pouca abstração (Boruchovitch, 1999). Entretanto, quando se trata de estratégias metacognitivas, surpreendeu o fato dos alunos mais novos terem alcançado uma pontuação maior, visto que tais estratégias demandam maior sensibilidade do aluno, na medida em que este tem que perceber quando não entendeu um conteúdo, um processo de maior complexidade. Contudo, crianças mais novas, apesar de empregarem estratégias menos sofisticadas, também são capazes de monitorar suas dificuldades e, por essa razão, podem ser sujeitos de uma intervenção com a finalidade de aperfeiçoar o uso de suas estratégias, bem como adquirir um novo repertório (Cruvinel, 2003).

No tocante aos anos escolares, os dados indicam que os alunos do $6^{\circ}$ ano obtiveram a melhor pontuação na escala. Tal dado também foi encontrado por Oliveira (2008), que, em sua pesquisa, verificou que, quanto mais avançam nos níveis de escolarização, menos os estudantes pontuam na escala. Quando se analisou as subescalas, percebeu-se que os alunos do $9^{\circ}$ ano obtiveram uma pontuação maior que os demais na subescala de estratégias metacognitivas, o que era esperado, visto que tais estratégias desenvolvem-se à medida que os sujeitos avançam no processo de escolarização e melhoram a percepção acerca do seu próprio processo de aprendizagem. De acordo com Costa (2000), à medida que o sujeito avança nos anos escolares e encontra tarefas mais complexas, precisa fazer uso de estratégias de aprendizagem cada vez mais sofisticadas.

No que se refere ao tipo de escola, acreditava-se, inicialmente, que os estudantes de escolas particulares, por terem mais acesso à informação e às estratégias de aprendizagem e possuírem um índice de repetência inferior aos alunos das escolas públicas, alcançariam escores mais elevados. Tal fato foi comprovado e os alunos das escolas privadas obtiveram uma pontuação mais alta na escala. Contudo, esse achado não corrobora os dados de Oliveira (2008), que, curiosamente, não encontrou diferença entre esses grupos.

Diante disso, pode-se pensar que os estados afetivos e motivacionais influenciam o sucesso e o insucesso 
escolar (Silva \& Sá, 1997). Cruvinel e Boruchovitch (2004) corroboram com esse pensamento e atentam que as estratégias de aprendizagem, apesar de serem importantes para a aprendizagem efetiva, não são suficientes para o sucesso acadêmico, uma vez que diversas variáveis psicológicas e motivacionais são determinantes no uso efetivo dessas estratégias.

Além disso, evidencia-se que, quando analisadas algumas palavras/expressões utilizadas na escala, existem sutis diferenças regionais. A exemplo disto, pode-se citar o item 4 da escala, no qual temos a palavra "lousa", bastante utilizada por escolares da região Sudeste e pouco usada por estudantes da região na qual a pesquisa se efetivou, que, ao invés dessa, utilizam a palavra "quadro-negro" ou simplesmente "quadro". Outras expressões que, apesar de não terem gerado dúvidas nos estudantes quanto ao seu significado, também se mostraram diferentes foram "dever de casa" e "lição de casa". Estas expressões são utilizadas na escala de estratégias de aprendizagem e, apesar de os alunos conhecerem e entenderem o seu significado, utilizam com mais frequência as expressões "tarefa de casa" e "atividade de casa" para se referirem a atividades que devem ser realizadas em casa. Diante disso, sugere-se que, para amostras da região Nordeste, estas palavras/expressões sejam alteradas com o objetivo de se obter uma melhor clareza nas questões.

De forma geral, os resultados demonstram que fatores como ano escolar, idade, sexo e repetência afetam o uso e o nível de desenvolvimento de algumas estratégias de aprendizagem. Contudo, uma limitação deste estudo é que não fizeram parte da amostra estudantes de todas as séries do Ensino Fundamental. Além disso, recomenda-se que pesquisas longitudinais e com outras medidas de comparação, como, por exemplo, a percepção do professor sobre o desempenho do aluno e notas escolares, sejam realizadas.

\section{Referências}

Almeida, L. S. (2002). Facilitar a aprendizagem: ajudar os alunos a aprender e a pensar. Psicologia Escolar e Educacional, 6(2), 155165.

Boruchovitch, E. (1999). Estratégias de aprendizagem e desempenho escolar: considerações para a prática educacional. Psicologia: reflexão e crítica, 12(2), 361-376.

Boruchovitch, E. (2001). Algumas estratégias de compreensão em leitura de alunos do Ensino Fundamental. Psicologia Escolar e Educacional, 5(1), 19-25.

Boruchovitch, E., Santos, A. A. A., Costa, E. R., Neves, E. R. C., Cruvinel, M., Primi, R., \& Guimarães, S. E. R. (2006). A construção de uma Escala de Estratégias de Aprendizagem para Alunos do Ensino Fundamental. Psicologia: teoria e pesquisa, 22(3), 297304.
Boruchovitch, E., Oliveira, K. L., \& Santos, A. A. A. (2007). Propriedades psicométricas de uma Escala de Estratégias de Aprendizagem para Alunos do Ensino Fundamental. III Congresso Brasileiro de Avaliação Psicológica [CD-ROM]. Paraíba: Instituto Brasileiro de Avaliação Psicológica.

Cerqueira, T. C. S. (2000). Estilos de aprendizagem em universitários. Tese de Doutorado, Faculdade de Educação, Universidade Estadual de Campinas, Campinas, São Paulo.

Costa, E. R. (2000). Estratégias de aprendizagem e a ansiedade de alunos do ensino fundamental: implicações para a prática educacional. Dissertação de Mestrado, Faculdade de Educação, Universidade Estadual de Campinas, Campinas, São Paulo.

Cruvinel, M. (2003). Depressão infantil, rendimento escolar e estratégias de aprendizagem em alunos do ensino fundamental. Dissertação de mestrado, Faculdade de Educação, Universidade Estadual de Campinas, Campinas, São Paulo.

Cruvinel, M., \& Boruchovitch, E. (2004). Sintomas depressivos, estratégias de aprendizagem e rendimento escolar de alunos do ensino fundamental. Psicologia em estudo, 9(3), 369-378.

Dansereau, D. F., Collins, K. W., McDonald, B. A., Holley, C. D., Garland, J., Diekhoff, G., \& Evans, S. H. (1979). Development and evaluation of a learning strategy training program. Journal of Educational Psychology, 71(1), 64-73.

Dembo, M. H. (1994). Applying educational psychology. New York: Longman Publishing Group.

Gomes, M. A. M. (2002). Aprendizagem auto-regulada em leitura numa perspectiva de jogos de regras. Dissertação de mestrado, Faculdade de educação, Universidade Estadual de Campinas, Campinas, São Paulo.

Muneiro, M. L. (2008). Estratégias de aprendizagem de alunos do ensino superior. Dissertação de mestrado, Faculdade de educação, Universidade Estadual de Campinas, Campinas, São Paulo.

Oliveira, K. L. (2008). Escala de estratégias de aprendizagem para o ensino fundamental: análise de suas propriedades psicométricas. Tese de Doutorado, Faculdade de Educação, Universidade Estadual de Campinas. Campinas, São Paulo.

Pintrich, P. R., Smith, D. A. F., Garcia, T., \& McKeachie, W. J. (1991). A manual for the use of the Motivated Strategies for Learning Questionnaire (MSLQ). Ann Arbor, Michigan: National Center for Research to Improve Postsecondary Teaching and Learning.

Pozo, J. I. (1996). Estratégias de aprendizagem. Em C. Coll, J. Palácios \& A. Marchesi (Orgs.), Desenvolvimento psicológico e educação: psicologia da educação (pp. 176-197). Porto Alegre: Artes Médicas. 
Pozo, J. I., Monereo, C., \& Castelló, M. (2004). O uso estratégico do conhecimento. Em C. Coll, A. Marchesi \& J. Palacios (Orgs.), Desenvolvimento psicológico e educação (pp. 145-160). Porto Alegre: Artes Médicas.

Silva, A. L., \& Sá, I. (1997). Saber estudar e estudar para saber. Coleção Ciências da Educação. Porto, Portugal: Porto Editora.
Weinstein, C. E., \& Palmer, D. R. (2002). Learning and Study Strategies Inventory (LASSI): user's manual (2a edição). Clearwater, Florida: $\mathrm{H}$ \& $\mathrm{H}$ Publishing Company.

Zimmerman, B. J., \& Martinez-Pons, M. (1986). Development of a structured interview for assessing student use of self-regulated learning strategies. American Educational Research Journal, 23, 614-628.

Recebido em: 15/04/2010

Reformulado em: 16/11/2010

Aprovado em: 08/06/2011

\section{Sobre as autoras}

\section{Manuela Ramos Caldas Lins}

Universidade Federal do Rio Grande do Norte, Natal-RN.

\section{Monilly Ramos Araujo}

Centro Universitário de João Pessoa, João Pessoa-PB.

Carla Alexandra da Silva Moita Minervino (carlamoita@globo.com) Universidade Federal da Paraíba, João Pessoa-PB.

\section{Correspondência}

\section{Carla Alexandra da Silva Moita Minervino}

Universidade Federal da Paraíba

Cidade Universitária - João Pessoa - PB -Brasil - CEP: 58059-900 